\title{
Le vent du changement souffle toujours
}

\author{
par Patrick Fitch
}

$\mathrm{I}_{\mathrm{m}}^{\mathrm{r}}$ y a deux ans, dans mon premier commentaire en tant que membre de l'équipe présidentielle de la Société canadienne des pharmaciens d'hôpitaux (SCPH) (J. Can. Pharm. Hosp. 2016; 69[5]:431) je me suis exprimé sur certains changements qui étaient intervenus peu auparavant au sein de notre profession et de la Société. En entamant ma dernière année à titre de membre de la direction de la SCPH, j'ai pensé qu'il serait opportun de me pencher à nouveau sur ce thème. D'importants changements se produiront à la SCPH et bon nombre d'entre eux influenceront la direction de la Société à l'avenir.

Le plus important est celui qui touche la direction. Le 31 décembre 2018, après 15 ans à la barre de la SCPH, la directrice générale, Myrella Roy, a cédé sa place à Jody Ciufo, qui a officiellement repris les rênes de la Société le 2 janvier 2019. Jody a une vaste expérience de la gestion associative et elle est prête à relever le défi que représente la direction de la SCPH dans les années à venir.

Lorsque Myrella Roy a annoncé son départ, le conseil a pensé que le moment était venu de mettre à jour le pendant anglais du titre du poste le plus élevé dans la direction de la SCPH. Or, aucun changement n'a été apporté au titre du poste qui, en français, demeure celui de directrice générale. Peut-être vous demandez-vous pourquoi le conseil a décidé de modifier le titre du poste le plus élevé de notre organisme. Historiquement, les cadres supérieurs des organismes sans but lucratif avaient le titre de "executive director ". Or, au cours des trois dernières décennies, bon nombre d'organismes ont décidé d'adopter le titre de "chief executive officer" pour se calquer sur le monde des affaires. Cependant, ce changement n'a pas lieu pour l'équivalent français, donc Jody conservera le titre directrice générale.

L'actuel plan stratégique de la SCPH arrivera à terme en 2020 et le travail d'élaboration du prochain plan est déjà amorcé. Le conseil se réunira en octobre 2019 pour participer à un atelier de planification d'une journée. Pour préparer cette rencontre, il s'est engagé à faire une évaluation stratégique de l'ensemble des programmes et services de la SCPH en gardant à l'esprit deux objectifs : guider le processus de planification stratégique et cibler les changements à apporter aux programmes et services qui feront de la SCPH un organisme plus fort.

Léquipe de liaison interne n'a pas chômé non plus. Lorsque nous avons pris conscience que nous n'atteindrions pas les cibles d'adhésion du plan stratégique actuel, un groupe de travail composé de membres du conseil, du comité d'adhésion et de présidents de sections a été mis en place pour élaborer des stratégies de recrutement et de fidélisation. Ces stratégies ont été approuvées par le conseil en avril 2018. Depuis lors, elles sont prioritaires, des groupes de travail supplémentaires ont donc été mis sur pied pour développer des activités de mise en œuvre.

Le conseil a aussi longuement étudié l'avenir de la Conférence sur la pratique professionnelle et un groupe de travail a été mis sur pied afin de trouver le moyen de la revitaliser au moment de célébrer son $50^{\mathrm{e}}$ anniversaire. Le travail d'évaluation de l'ensemble des programmes de formation de la SCPH se poursuit afin que soient offertes à nos membres des formations de qualité à la hauteur de leurs attentes .

Le programme de reconnaissance a lui aussi fait l'objet d'un examen et des changements s'annoncent, tant pour le programme national que pour celui des sections. L'objectif vise à rationaliser les propositions de prix pour éviter les redondances.

Au moment où la SCPH évalue ses programmes et services, nous sollicitons de plusieurs façons des commentaires de la part de nos membres : enquêtes, sondages, médias sociaux. J'invite tous les membres à nous faire part de leurs opinions le moment venu.

Je crois qu'une période stimulante s'amorce pour la SCPH et j'ai hâte de voir ce qui nous attend.

[Traduction par l'éditeur]

Patrick Fitch, B. S. P., A. C. P. R., est président sortant et agent de liaison interne pour la Société canadienne des pharmaciens d'hôpitaux. 